CONGENITAL HEART DISEASE

\title{
Structural and functional abnormalities of large arteries in the Turner syndrome
}

\author{
J-P Baguet, S Douchin, H Pierre, A-M Rossignol, M Bost, J-M Mallion
}

Heart 2005:91:1442-1446. doi: 10.1136/hrt.2004.048371

See end of article for authors' affiliations

.....................

Correspondence to: Dr Jean-Philippe Baguet, Service de Cardiologie et Hypertension Artérielle, CHU A Michallon, BP 217 , 38043 Grenoble Cedex 09, France; JPBaguet@ chu-grenoble.fr

Accepted 2 February 2005 Published Online First 10 March 2005
Objective: To analyse the structural and functional abnormalities in the large arteries in women with the Turner syndrome.

Methods: Aortic stiffness (assessed by means of the carotid femoral pulse wave velocity), level of amplification of the carotid pressure wave (by applanation tonometry), and carotid remodelling (by high resolution ultrasound) were studied in women with the Turner syndrome. Clinical and ambulatory blood pressures were taken into account in the analysis. Thus, 24 patients with the Turner syndrome and 25 healthy female subjects matched for age were studied.

Results: Women with the Turner syndrome had a higher augmentation index than the controls (Turner, mean (SD) $0.04(0.14) v$ controls, $-0.14(0.13), p<0.001)$ but a lower peripheral pulse pressure (39 (8) $\mathrm{mm} \mathrm{Hg} \vee 47$ (11) mm Hg, $\mathrm{p}=0.010$ in the clinic; 44 (5) mm Hg $\vee 47$ (6) mm Hg, $\mathrm{p}=0.036$ during the 24 hour ambulatory recording). The luminal diameter of the common carotid artery and the carotid-femoral pulse wave velocity were similar in the two groups, whereas carotid intima-media thickness tended to be higher in women with the Turner syndrome $(0.53(0.06) \mathrm{mm} v 0.50(0.05) \mathrm{mm}$, $p=0.06$ ). After correction for body surface area, carotid intima-media thickness and pulse wave velocity were higher in women with the Turner syndrome.

Conclusions: Vascular abnormalities observed in the Turner syndrome are implicated in the origin of the cardiovascular complications that occur in this syndrome. These abnormalities are morphological but also functional. An increase in the augmentation index can be explained in part by the short height of these patients.
$\mathrm{T}$ he Turner syndrome is associated with a chromosomal defect (monosomy XO or structural abnormality of one of the $\mathrm{X}$ chromosomes) that affects roughly $\mathrm{l}$ in 3000 newborn girls with a characteristic phenotype of growth retardation and gonadal dysgenesis leading to failure of puberty and sterility.

Congenital and acquired cardiovascular abnormalities are often associated with this syndrome and are found in about $30 \%$ of these patients. ${ }^{1-5}$ The congenital cardiac abnormalities observed are essentially a bicuspid aortic valve, aortic coarctation, aortic kinking, mitral valve prolapse, and aneurysmal dilatation of the ascending aorta.

Among the cardiac and arterial abnormalities, it is essentially the aortic conditions that favour the development of acute complications that can be fatal, such as aortic dissection and rupture of an aneurysm in the ascending aorta. Some authors have found in women with the Turner syndrome histological evidence of cystic necrosis of the media in the ruptured aortic wall suggesting a constitutional tissue dysplasia with cystic medial necrosis similar to that found in patients with Marfan's syndrome. ${ }^{67}$ However, these lesions are not constant. Other factors, such as an increased blood pressure (BP), also play a part in the development of aortic wall lesions. Indeed, more than a quarter of women with the Turner syndrome have hypertension and $50 \%$ of them have an abnormal BP circadian rhythm. ${ }^{8-11}$

The pathological effects in arterial territories other than the aorta in the Turner syndrome are not well known. Thus, we set out to analyse aortic stiffness, level of amplification of the carotid pressure wave, and carotid remodelling in patients with this syndrome.

\section{METHODS}

\section{Population}

This study was carried out in 24 women with genetically proven Turner syndrome $(45, \mathrm{X} \quad(\mathrm{n}=12) ; 45, \mathrm{X} / 46, \mathrm{XX}$ $(\mathrm{n}=1) ; 45, X / 46, X i X q(\mathrm{n}=4) ; 46, X i X q(\mathrm{n}=2) ; 46, X X /$ $46, X i X q(\mathrm{n}=1) ; 46, \mathrm{XXq}-(\mathrm{n}=1) ; 45, \mathrm{X} / 46, \mathrm{XrX}(\mathrm{n}=1)$; 45, $\mathrm{X} / 46, \mathrm{XY}(\mathrm{n}=1) ; 45, \mathrm{X} / 46, \mathrm{XY} / 47, \mathrm{XY}+\operatorname{mar}(\mathrm{n}=1))$ who were followed up in the Paediatrics or Cardiology Departments at Grenoble University Hospital. The mean (SD) age was 17.3 (6.8) years (range 7-30 years). Patients were recruited consecutively by written invitation for consultation. This study complies with the Declaration of Helsinki and was approved by the ethics committee of the Grenoble University Hospital. All the subjects gave informed consent. Six of the patients had previously diagnosed cardiovascular conditions (one had Shone syndrome operated on at the age of 8 years, one had aortic coarctation operated on at the age of 3 years, two had aortic kinking, and two had essential hypertension). Twelve of the patients had hormonal treatment with an oestrogen-progestogen combination, three others had discontinued this treatment, and the remaining nine did not receive the treatment because of age (eight patients were too young) or spontaneous puberty development (one patient). Twelve had growth hormone treatment and six had stopped this hormone, as they had reached their final height. Two patients were treated for hypertension, one with an angiotensin conversion enzyme inhibitor and one with a calcium channel blocker. Twenty five healthy female subjects, recruited from outside the hospital (students) with a mean age of 19 (4.3) years (range 11-26 years), constituted the control group and were matched to the patients for age. Nine of the controls were taking a combined oestrogen-progestogen contraceptive treatment. Only two patients and none of the controls were

Abbreviations: $\mathrm{Al}$, augmentation index; $\mathrm{BP}$, blood pressure; $\mathrm{BSA}$, body surface area; CSA, cross sectional area; DBP, diastolic blood pressure; $H R$, heart rate; IMT, intima-media thickness; LV, left ventricular; PP, pulse pressure; PWV, pulse wave velocity; SBP, systolic blood pressure 
smokers. All investigations were carried out on each patient over a period of a day.

\section{$B P$ and heart rate measurements}

Clinic BP and heart rate (HR) were measured on three occasions according to European Society of HypertensionEuropean Society of Cardiology guidelines. ${ }^{12}$ Non-invasive ambulatory BP monitoring was carried out with a Spacelabs 90207 monitor (Spacelabs International, Redmond, Washington, USA). The measurements were made every 15 minutes over 24 hours. The following parameters were studied over the 24 hours and over daytime and night time: mean systolic BP (SBP), diastolic BP (DBP), mean BP, pulse pressure $((\mathrm{PP})$ defined as $\mathrm{PP}=\mathrm{SBP}-\mathrm{DBP})$, and $\mathrm{HR}$. SBP $>260 \mathrm{~mm} \mathrm{Hg}$ and $<70 \mathrm{~mm} \mathrm{Hg}$ and $\mathrm{DBP}>150 \mathrm{~mm} \mathrm{Hg}$ and $<40 \mathrm{~mm} \mathrm{Hg}$ were automatically eliminated. Criteria to validate ambulatory BP monitoring recording were cuff size adapted to the arm circumference, control of the standardisation of the device, duration of the recording $\geqslant 24$ hours with more than 48 valid measurements, and missing data in a less than two consecutive hour bracket.

\section{Carotid tonometry}

The carotid pressure wave was calculated by applanation tonometry (Millar SPT-301, Millar Instruments, Houston, Texas, USA) with precalibration ( $1 \mathrm{~mm} \mathrm{Hg}=1 \mathrm{mV}$ ) by a preamplifier coupled to the recording of the digital arterial BP. Pressure waves were recorded at $500 \mathrm{~Hz}$ by a specific program. SBP and DBP were measured at the beginning of the examination with a mercury sphygmomanometer on the arm. These values were used for calibration with the method of surfaces, based on the assumption that brachial mean BP remains constant over the length of the arterial tree. Brachial mean BP was assigned the area under the carotid waveform, and brachial DBP was assigned the automatically determined end diastolic point on the carotid waveform. The carotid augmentation index (AI) was calculated automatically by a dedicated program for analysis of pressure curves. The AI was defined as the difference in BP between the second and the first peak of the carotid pressure wave divided by the carotid PP. ${ }^{13}$

\section{Carotid-femoral pulse wave velocity}

To determinate the carotid-femoral pulse wave velocity (PWV), two pulse transducers were fixed on the skin over the right common carotid and femoral arteries. The time delay was measured with a Complior device (Artech Medical, Pantin, France) between the feet of simultaneously recorded pulse waves and averaged over 10 consecutive cycles. The carotid-femoral PWV was calculated as the distance between the arterial sites divided by the time delay.

\section{Carotid ultrasonography}

B mode ultrasonography was performed with a Sonos 2500 (Hewlett Packard, Santa Clara, California, USA) machine. The method has been previously published.${ }^{14}$ Both common carotid arteries were studied consecutively. The images were recorded in end diastole and then stored on an optical disc for subsequent analysis by a specific program (TIMC Laboratory, CHU Grenoble, France). The values of intima-media thickness (IMT) and of luminal diameter for any subject were the mean values for the two carotid arteries. The arterial geometry was studied from the cross sectional area (CSA), defined as $\mathrm{CSA}=\pi \times(\mathrm{IMTd}+(\mathrm{Dd} / 2))^{2}-\pi \times(\mathrm{Dd} / 2)^{2}$, where IMTd is IMT in end diastole and Dd is luminal diameter in end diastole. Carotid IMT, luminal diameter, and CSA were calculated before and after correction for height.
Table 1 General, biological, and haemodynamic characteristics of patients with the Turner syndrome and of controls

\begin{tabular}{|c|c|c|c|}
\hline Variable & $\begin{array}{l}\text { Turner } \\
\text { syndrome } \\
(\mathrm{n}=24)\end{array}$ & $\begin{array}{l}\text { Controls } \\
(n=25)\end{array}$ & p Value \\
\hline Age (years) & $17.3(6.8)$ & $19.0(4.3)$ & 0.32 \\
\hline Height $(\mathrm{cm})$ & $141(15)$ & $163(8)$ & $<0.001$ \\
\hline Weight (kg) & $44(14)$ & $55(9)$ & 0.003 \\
\hline BMI $\left(\mathrm{kg} / \mathrm{m}^{2}\right)$ & $21.7(4.1)$ & $20.3(2.7)$ & 0.17 \\
\hline BSA $\left(\mathrm{m}^{2}\right)$ & $1.29(0.26)$ & $1.58(0.16)$ & $<0.001$ \\
\hline Total cholesterol (mmol/l) & $4.89(0.76)$ & $4.85(0.80)$ & 0.85 \\
\hline Triglyceride (mmol/l) & $1.20(1.05)$ & $0.87(0.38)$ & 0.14 \\
\hline $\mathrm{HDL}$ cholesterol (mmol/l) & $1.60(0.48)$ & $1.62(0.31)$ & 0.89 \\
\hline LDL cholesterol (mmol/I) & $2.74(0.68)$ & $2.83(0.63)$ & 0.62 \\
\hline Clinic SBP $(\mathrm{mm} \mathrm{Hg})$ & $119(12)$ & $119(9)$ & 0.94 \\
\hline Clinic DBP (mm Hg) & $80(12)$ & $72(6)$ & 0.012 \\
\hline Clinic MBP (mm Hg) & $93(12)$ & $88(5)$ & 0.07 \\
\hline Clinic PP $(\mathrm{mm} \mathrm{Hg})$ & $39(8)$ & 47 (11) & 0.010 \\
\hline ABPM SBP (mm Hg) & $117(9)$ & $118(7)$ & 0.95 \\
\hline ABPM DBP $(\mathrm{mm} \mathrm{Hg})$ & $74(9)$ & $70(5)$ & 0.13 \\
\hline ABPM MBP $(\mathrm{mm} \mathrm{Hg})$ & $89(9)$ & $86(5)$ & 0.19 \\
\hline ABPM PP $(\mathrm{mm} \mathrm{Hg})$ & $44(5)$ & $47(6)$ & 0.036 \\
\hline Carotid PP (mm Hg) & $45(7)$ & $48(9)$ & 0.19 \\
\hline Clinic HR (beats/min) & $86(13)$ & 74 (12) & 0.002 \\
\hline ABPM HR (beats/min) & $90(9)$ & $77(8)$ & $<0.001$ \\
\hline \multicolumn{4}{|c|}{$\begin{array}{l}\text { Data are mean (SD). } \\
\text { ABPM, ambulatory blood pressure measurement; BMI, body mass index; } \\
\text { BSA, body surface area; DBP, diastolic blood pressure; HDL, high density } \\
\text { lipoprotein; HR, heart rate; LDL, low density lipoprotein; MBP, mean } \\
\text { blood pressure; PP, pulse pressure; SBP, systolic blood pressure. }\end{array}$} \\
\hline
\end{tabular}

\section{Cardiac ultrasound}

The echocardiogram was recorded by the same machine as that used for the carotid ultrasound (2.5 $\mathrm{MHz}$ probe). The examination was performed in TM mode with two dimensional guidance in the long axis of the left parasternal view. The measures of the interventricular septum and the left ventricular (LV) posterior wall were calculated in end diastole over five consecutive cycles. LV internal diameter was measured in end diastole and end systole to determine LV shortening fraction. Aortic diameter was calculated in two dimensional mode at the level of the Valsalva sinus (left parasternal view) and of the arch (suprasternal view).

\section{Biological parameters}

Fasting total plasma cholesterol (enzymatic colorimetry, normal 4.62-7.04 mmol/l), triglyceride (enzymatic colorimetry, normal $0.64-2.60 \mathrm{mmol} / \mathrm{l})$, high density lipoprotein cholesterol (enzymatic colorimetry, normal $1.00-1.78 \mathrm{mmol} /$ 1), and low density lipoprotein cholesterol (Friedewald formula, normal $2.61-4.67 \mathrm{mmol} / \mathrm{l}$ ) were measured for all subjects.

\section{Statistical analysis}

Data were analysed with SPSS software (SPSS Inc, Chicago, Illinois, USA). After verification of the normality of distribution of the variables, mean (SD) were calculated. The groups were compared by an independent $t$ test. Relations between the continuous variables were evaluated by Pearson's correlation coefficient ( $\mathrm{p}<0.05$ was considered significant). Multivariate analysis was performed, separately for each group, by a stepwise regression taking into account the two variables that were most correlated with the dependent variables (Student-Newman-Keuls test).

\section{RESULTS}

Table 1 gives general, biological, and haemodynamic characteristics of the patients and controls. Patients with the Turner syndrome had a shorter height but the same body mass index as the controls. Lipid profiles did not differ 


\begin{tabular}{|c|c|c|c|}
\hline Variable & Turner syndrome $(n=24)$ & Controls ( $n=25$ ) & $\mathrm{p}$ Value \\
\hline Al & $0.04(0.14)$ & $-0.14(0.13)$ & $<0.001$ \\
\hline $\mathrm{Al} /$ clinic $\mathrm{HR}\left((\text { beats } / \mathrm{min})^{-1}\right)$ & $0.0004(0.002)$ & $-0.0019(0.002)$ & $<0.001$ \\
\hline $\mathrm{Al} / \mathrm{BSA}\left(\mathrm{m}^{-2}\right)$ & $0.026(0.108)$ & $-0.089(0.086)$ & $<0.001$ \\
\hline $\mathrm{PWV}(\mathrm{m} / \mathrm{s})$ & $6.53(1.28)$ & 7.10 (1.08) & 0.10 \\
\hline PWV/HR (m/s.beats/min) & $0.08(0.02)$ & $0.10(0.02)$ & 0.002 \\
\hline $\mathrm{PWV} / \mathrm{BSA}\left(\mathrm{m} / \mathrm{s} / \mathrm{m}^{2}\right)$ & $5.19(1.19)$ & $4.51(0.62)$ & 0.016 \\
\hline Carotid IMT (mm) & $0.53(0.06)$ & $0.50(0.04)$ & 0.06 \\
\hline Carotid IMT/H $(\mathrm{mm} / \mathrm{m})$ & $0.38(0.10)$ & $0.31(0.08)$ & $<0.001$ \\
\hline Carotid IMT/BSA $\left(\mathrm{mm} / \mathrm{m}^{2}\right)$ & $0.43(0.12)$ & $0.32(0.04)$ & $<0.001$ \\
\hline Carotid diameter $(\mathrm{mm})$ & $5.17(0.54)$ & $5.26(0.36)$ & 0.50 \\
\hline Carotid diameter $/ \mathrm{H}(\mathrm{mm} / \mathrm{m})$ & $3.68(0.41)$ & $3.22(0.24)$ & $<0.001$ \\
\hline Carotid diameter $/$ BSA $\left(\mathrm{mm} / \mathrm{m}^{2}\right)$ & $4.13(0.79)$ & $3.36(0.34)$ & $<0.001$ \\
\hline Carotid CSA $\left(\mathrm{mm}^{2}\right)$ & $9.45(1.54)$ & $9.03(1.19)$ & 0.29 \\
\hline Carotid CSA/H $\left(\mathrm{mm}^{2} / \mathrm{m}\right)$ & $6.75(1.21)$ & $5.53(0.73)$ & $<0.001$ \\
\hline Carotid CSA/BSA $\left(\mathrm{mm}^{2} / \mathrm{m}^{2}\right)$ & $7.57(1.95)$ & $5.75(0.73)$ & $<0.001$ \\
\hline LV shortening fraction (\%) & $40(5)$ & $39(4)$ & 0.89 \\
\hline \multicolumn{4}{|l|}{ Aortic diameter $(\mathrm{mm})$} \\
\hline Valsalva sinus $(\mathrm{mm})$ & $25.8(2.5)$ & $24.1(4.1)$ & 0.08 \\
\hline Valsalva sinus/BSA $\left(\mathrm{mm} / \mathrm{m}^{2}\right)$ & $19.7(2.8)$ & $15.5(1.5)$ & $<0.001$ \\
\hline Arch $(\mathrm{mm})$ & $16.9(2.3)$ & $14.7(2.0)$ & 0.001 \\
\hline $\operatorname{Arch} / \mathrm{BSA}\left(\mathrm{mm} / \mathrm{m}^{2}\right)$ & $12.9(2.3)$ & $9.6(0.9)$ & $<0.001$ \\
\hline
\end{tabular}

significantly between the groups. One third of the patients (eight of 24) had clinical hypertension (defined as clinic SBP $\geqslant 140 \mathrm{~mm} \mathrm{Hg}$ or DBP $\geqslant 90 \mathrm{~mm} \mathrm{Hg}$ ). This was predominantly ( seven of eight $(87 \%)$ ) isolated diastolic hypertension. All the controls were normotensive in clinic. Clinic and 24 hour SBP and mean BP were identical in the two groups even though the clinical DBP was higher in the group with the Turner syndrome. Clinic and 24 hour PP were lower in women with the Turner syndrome. Carotid PP was identical in both groups, whereas HR was greater in women with the Turner syndrome both in the clinic and in ambulatory recordings.

Table 2 shows the arterial and cardiac measurements. The carotid to femoral PWV was not different between the two groups. After correction for HR, PWV was lower in women with the Turner syndrome. After correction for body surface area (BSA), PWV was higher in patients. The AI was greater in patients with the Turner syndrome even before taking into account the BSA (fig 1). Carotid luminal diameter and CSA were identical in the two groups, whereas carotid IMT tended to be higher in women with the Turner syndrome. When height or BSA was taken into account, all these parameters were higher in women with the Turner syndrome. In the patients, aortic diameter tended to be higher at the level of the Valsalva sinus and was higher at the aortic arch than in controls. After correction for BSA, aortic diameters were higher in women with the Turner syndrome. LV function was similar in both groups.

Patients receiving or having received growth hormone treatment ( 18 of $24(75 \%)$ ) were younger than women who were not treated ( $14.9(4.4)$ and $24.5(8.0)$ years, respectively, $\mathrm{p}<0.05)$. The prevalence of hypertension was similar in patients receiving (six of 18) and not receiving growth hormone (two of six). There was no difference between patients who were and patients who were not taking hormonal replacement therapy in terms of the various BP parameters.

Univariate analysis of data for women with the Turner syndrome showed that age and clinic SBP were correlated with carotid IMT/height $(p=0.01$ and $p=0.028$, respectively), carotid IMT/BSA ( $p=0.01$ and $p<0.001$, respectively), $\operatorname{PWV}(\mathrm{p}=0.001$ and $\mathrm{p}=0.004$, respectively), AI $(\mathrm{p}=0.001$ and $\mathrm{p}=0.049$, respectively), and AI/BSA $(\mathrm{p}<0.001$ and $\mathrm{p}=0.048$, respectively). Clinic DBP was correlated with AI $(p=0.014)$, AI/BSA $(p=0.011)$, IMT/ BSA $(p<0.001)$, and PWV $(p=0.016)$. Height was not correlated with AI, PWV, or carotid IMT.

Table 3 shows the results of multivariate analysis of these data. In women with the Turner syndrome, the single parameter retained in the prediction models of AI, AI/BSA, PWV, and size indexed carotid IMT was age. In the patients, clinic DBP was independently correlated with carotid IMT/ BSA. In the control subjects, PWV was correlated with age independently of clinic DBP and PP measured by tonometry.

In women with the Turner syndrome, the diameter of the Valsalva sinus was positively correlated with clinic BP $(p<0.01$ for both clinic SBP and DBP). Likewise, the diameter of the aortic arch was correlated with clinic BP $(p<0.05$ for both clinic SBP and DBP). These significant relations persisted after correction for BSA. AI tended to be related to the diameter of the aortic arch $(p=0.07)$.

\section{DISCUSSION}

The present study has characterised the morphological and functional characteristics of the large arteries in a population of 24 women with the Turner syndrome compared with 25 control subjects matched for age. We have shown that patients with the Turner syndrome have a higher AI of the carotid pressure wave than controls and confirmed the importance of height in the determination of this index. Peripheral PP, but not central PP, was lower in patients with the Turner syndrome. After correction for BSA, PWV was higher in women with the Turner syndrome. Carotid IMT, luminal diameter, and CSA were identical in the two groups. However, these carotid parameters were higher in women with the Turner syndrome when corrected for height or BSA. Aortic diameters were higher in patients, more clearly after correction for BSA.

The Turner syndrome is often associated with cardiac and aortic malformations. Acute complications of this syndrome, which involve principally the aortic wall, may have critical consequences. The principal factors that can lead to such complications are hypertension and histological abnormalities in the vascular wall. Thus, hypertension favours the development of aortic wall lesions responsible for aortic haematomas or dissection. In our study, the prevalence of 

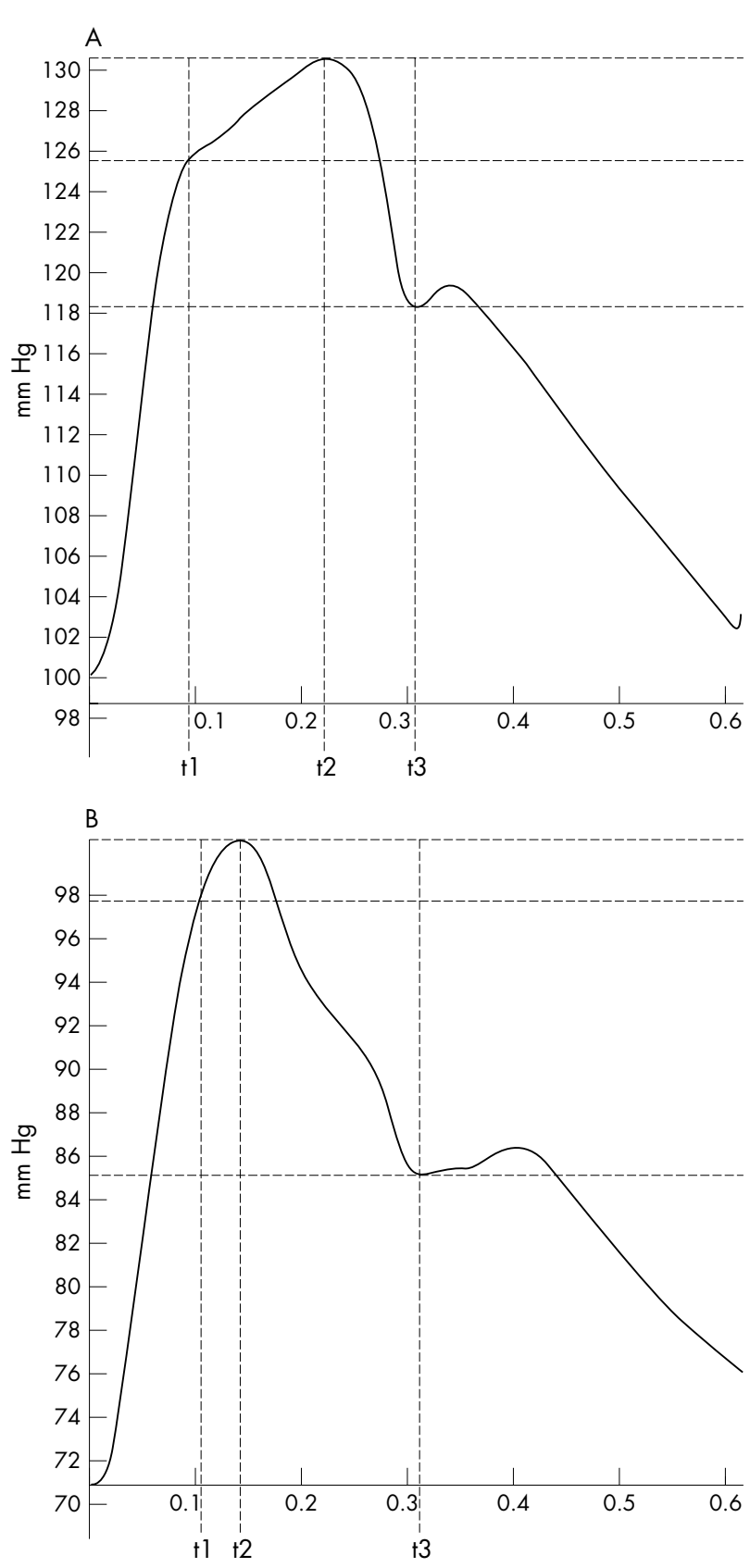

Figure 1 Typical carotid pulse wave (applanation tonometry) for (A) a woman with the Turner syndrome (augmentation index (Al) 19\%) and (B) a control subject (Al - 23\%). " $\mathrm{t}$ " is sampling time: $\mathrm{tl}$, time of the first peak; $\nmid 2$, time of the second peak; +3 , end systolic time.

clinical hypertension in patients with the Turner syndrome was increased, similar to that reported in the literature, being of the order of $30 \%$. In women with the Turner syndrome, the single parameter retained in the prediction models of AI, PWV, and size indexed IMT was age. Furthermore, some authors have found lesions of cystic media necrosis in the aortic walls of patients with the Turner syndrome who have aortic dissection. These lesions should suggest the presence of constitutional tissue dysplasia in this condition.

\section{Augmentation index}

An increase in the AI of the arterial pressure wave is classically related to a reduction in the reflection time of this wave. Basic haemodynamic studies have shown that the amplitude of the reflection waves depends not only on
Table 3 Multivariate analysis (sig T) of arterial parameters in patients with the Turner syndrome and in controls

\begin{tabular}{|c|c|c|}
\hline & Variables retained & Other variables tested \\
\hline \multicolumn{3}{|c|}{ Turner $(n=24)$} \\
\hline $\mathrm{Al}$ & Age: 0.0006 & $\begin{array}{l}\text { Clinic and ABPM DBP, clinic } \\
\text { SBP, PWV }\end{array}$ \\
\hline $\mathrm{Al} / \mathrm{BSA}$ & Age: 0.0005 & $\begin{array}{l}\text { Clinic and ABPM DBP, clinic } \\
\text { SBP, PWV }\end{array}$ \\
\hline PWV & Age: 0.0010 & $\begin{array}{l}\text { Clinic DBP and SBP, Al, Al/ } \\
\text { BSA, BMI }\end{array}$ \\
\hline IMT/H & Age: 0.0099 & Clinic DBP \\
\hline IMT/BSA & Clinic DBP: 0.0011 & Age, clinic SBP, PWV \\
\hline \multicolumn{3}{|c|}{ Controls ( $n=25$ ) } \\
\hline PWV & $\begin{array}{l}\text { Carotid PP: } 0.0027 \\
\text { Age: } 0.0357\end{array}$ & \\
\hline
\end{tabular}

arteriolar tone, peripheral resistance, distribution, and geometric changes in the arterial segment touched by the wave, but also on the height of the subjects and on their HR. ${ }^{15-17}$ In our study, women with the Turner syndrome had larger and earlier reflection waves than the controls. The multivariate analysis of our data showed that the only independent predictive factor of the AI in the patients was age. Height was not retained in the model partly because of the small size of the overall group.

In the study reported by Elsheik et al, ${ }^{11}$ there was no relation between the dilation of the aortic root in women with the Turner syndrome and the arterial function measured by AI. ${ }^{11}$ These data need to be confirmed because in that study $50 \%$ of the patients (mean age 29 years) had hypertension and should thus have had antihypertensive treatment. In women with the Turner syndrome, we found a trend only for a relation between aortic arch diameter and AI. As Elsheik et $a l^{11}$ also previously described, we found a positive and significant relation between aortic diameters and clinic BP, for both SBP and DBP.

\section{Pulse pressure}

In healthy subjects, PP is principally determined by the incident pressure wave. In theory, in the group of women with the Turner syndrome who have a raised AI, PP is principally determined by the amplitude of the late systolic peak related to the early reflection waves. However, we did not find a difference in SBP between the two groups, although DBP was higher in women with the Turner syndrome, thus explaining the lower PP in these patients. These data may be explained in part by the small number of subjects included in the study, but also by the young age of the patients, of whom two were treated for hypertension and two had been operated on for aortic coarctation. LV function being identical between the two groups, the reduced PP would also suggest that the overall elasticity of the large capacitance vessels is slightly improved or favourably adapted to maintain PWV within the normal range.

\section{Aortic stiffness}

In our study, carotid to femoral PWV tended to be lower in women with the Turner syndrome. After correction for HR, PWV was significantly lower in women with the Turner syndrome. This favours the idea of the arterial system being adapted to the haemodynamic conditions of this disease, in particular tachycardia. However, after correction for BSA, PWV was higher in women with the Turner syndrome. This confirms the role of height in PWV and is in agreement with the increase of AI and the carotid remodelling observed in the present study in women with the Turner syndrome. 


\section{Carotid remodelling}

To our knowledge, the walls of the common carotid arteries have never been studied by ultrasound in the Turner syndrome. We did not find a difference between the group of women with the Turner syndrome and the control group as regards luminal diameter and CSA of the carotid artery. On the other hand, carotid IMT tended to be higher in women with the Turner syndrome. When corrected for height or BSA, all these parameters are greater in women with the Turner syndrome. Although this index had not been clearly shown to be of interest at the carotid level, in particular in hypertension, it seemed interesting to study the carotid parameters after taking into account the short height of women with the Turner syndrome. ${ }^{18}$ These last data evoke but do not assert the presence of a carotid remodelling in the Turner syndrome.

\section{Effects of treatment}

The present study was not designed to find any difference between the two groups according to treatment (that is, growth hormone, antihypertensive drugs, or hormone replacement therapy). The effect of growth hormone treatment on the arterial wall has not been specifically studied in the Turner syndrome. This hormone, by increasing a patient's height, ought to decrease the amplitude and increase the delay in the reflection waves of arterial pressure. $\beta$ Blockers seem to be the antihypertensive drugs of choice in the Turner syndrome perhaps because of their effect on HR. The deficit in oestrogens in women with the Turner syndrome partly explains the increased cardiovascular risk in this population. Elsheikh et al $^{19}$ have shown that hormone replacement therapy has a beneficial effect on haemodynamic function in the large arteries of patients with the Turner syndrome. This result was attributed to an improvement in endothelial function. Likewise, Gravholt et $a l^{9}$ have found a beneficial effect of sex hormone treatment on BP. Thus, from a cardiovascular point of view, these data should justify treating women who have the Turner syndrome with growth hormone, hormone replacement therapy, and $\beta$ blockers if BP levels permit.

\section{Study limitations}

The number of subjects included in the present study seems to be small but this is due to the rarity of the Turner syndrome. Moreover, the population is quite heterogeneous, particularly concerning treatment, which sometimes makes subgroup analysis difficult to interpret. However, it is difficult to have a homogeneous population in this sort of disease where patients have been monitored and treated for a great many years.

The high frequency (eight of 24) of clinical hypertension found in women with the Turner syndrome may explain an important part of the findings in the present study. Indeed, the role of BP in large arteries is well known with hyperplasia and degeneration of the arterial wall. Moreover, six of the patients had previously diagnosed cardiovascular conditions including two patients with treated hypertension. Likewise, univariate analysis confirmed the relation between arterial parameters and BP variables in women with the Turner syndrome. However, even though the clinic DBP was higher in the group with the Turner syndrome, clinic mean BP and all the ambulatory BP monitoring measurements did not differ between the two groups. Moreover, in multivariate analysis, excepting the independent association between clinic DBP and carotid IMT/BSA, the relation between arterial parameters and BP data disappeared after taking into account all the parameters correlated with the vascular variables in patients and in controls.
In patients with aortic kinking, the distance between the carotid and femoral arteries, used to calculate the PWV, was measured without taking the aortic kinking into account. However, in clinical practice it is not possible to calculate this true distance accurately.

\section{Conclusion}

The vascular abnormalities found in the Turner syndrome appear to cause the cardiovascular complications found in this condition. These are morphological but also functional, with increase in the AI of the carotid pressure wave secondary to the short height and increased HR in these patients. The presence of carotid remodelling cannot be asserted in this condition, since it is only evident after correction for height or BSA. Prospective studies are necessary for a better understanding of this syndrome, especially to analyse its spontaneous evolution and to improve diagnosis of the cardiovascular abnormalities and medical treatment.

\section{Authors' affiliations}

J-P Baguet, H Pierre, J-M Mallion, Department of Cardiology and Hypertension, University Hospital, Grenoble, France

S Douchin, A-M Rossignol, M Bost, Department of Paediatrics, University Hospital, Grenoble, France

\section{REFERENCES}

1 Dawson FKL, Wright AM, Bakker B, et al. Cardiovascular evaluation in Turner syndrome: utility of MR imaging. Australas Radiol 1992;36:204-9.

2 Gotzsche CO, Krag-Olsen B, Nielsen J, et al. Prevalence of cardiovascular malformations and association with karyotypes in Turner's syndrome. Arch Dis Child 1994;71:433-6.

3 Mazzanti L, Cacciari E. Congenital heart disease in patients with Turner's syndrome. Italian study group for Turner syndrome (ISGTS). J Pediatr 1998; 133:688-92.

4 Sybert VP. Cardiovascular malformations and complications in Turner syndrome. Pediatrics 1998;101:E11.

5 Douchin S, Rossignol AM, Klein SK, et al. Cardiac malformations and vascular complications of Turner's syndrome. A prospective study of 26 patients. Arch Mal Coeur Vaiss 2000;93:565-70.

6 Palmer CG, Reichman A. Chromosomal and clinical findings in 110 females with Turner's syndrome. Hum Genet 1976:35:35-9.

7 Lin AE, Lippe BM, Geffner ME, et al. Aortic dilation, dissection, and rupture in patients with Turner's syndrome. J Pediatr 1986;109:820-6.

8 Gravholt CH, Juul S, Naeraa RW, et al. Morbidity in Turner syndrome. J Clin Epidemiol 1998;51:147-58

9 Gravholt CH, Naeraa RW, Nyholm B, et al. Glucose metabolism, lipid metabolism, and cardiovascular risk factors in adult Turner's syndrome. Diabetes Care 1998;21:1062-70.

10 Nathwani NC, Unwin R, Brook GD, et al. Blood pressure and Turner syndrome. Clin Endocrinol 2000;52:363-70.

11 Elsheikh M, Casadei B, Conway GS, et al. Hypertension is a major risk factor for aortic root dilation in women with Turner's syndrome. Clin Endocrinol 2001;54:69-73.

12 European Society of Hypertension, European Society of Cardiology. European Society of Hypertension-European Society of Cardiology guidelines for the management of arterial hypertension. Guidelines committee. $J$ Hypertens 2003;21:1011-53.

13 Nichols WW, O'Rourke M. McDonald's blood flow in arteries. In: Arnold E, eds. Theoretical, experimental and clinical principles, 4th ed. London: Arnold, 1998, 54-113, 201-22, 284-92, 347-401..

14 Baguet JP, Mallion JM, Moreau-Gaudry A, et al. Relationships between cardiovascular remodelling and the pulse pressure in never treated hypertension. J Hum Hypertens 2000;14:24-30.

15 London GM, Guerin AP, Pannier BM, et al. Body height as a determinant of carotid pulse contour in humans. J Hypertens Suppl 1992;10:S93-5.

16 Wilkinson IB, MacCallum H, Flint L, et al. The influence of heart rate on augmentation index and central arterial pressure in humans. J Physiol 2000;525:263-70.

17 Gatzka CD, Kingwell BA, Cameron JD, et al. Gender differences in the timing of arterial wave reflection beyond differences in body height. $J$ Hypertens 2001; 19:2197-203.

18 Baguet JP, Moreau-Gaudry A, Siché JP, et al. Carotid remodelling in essential hypertension: role of blood pressure, indexed parameters and association with cardiac mass and aortic stiffness. Clin Exp Hypertens 2000;22:717-29.

19 Elsheikh M, Bird R, Casadei B, et al. The effect of hormone replacement therapy on cardiovascular hemodynamics in women with Turner's syndrome. $J$ Clin Endocrinol Metab 2000;85:614-8. 